\title{
Clinical relevance of LINCO0152 and its variants in western Chinese tuberculosis patients
}

\author{
Jing Li ${ }^{1, *}$, Lijuan Wu ${ }^{2, *}$, Weihua Guo ${ }^{1}$, Juli Chen ${ }^{1}$, Xuejiao $\mathrm{Hu}^{2}$, Minjin Wang ${ }^{2}$, \\ Zhenzhen Zhao ${ }^{2}$ and Binwu Ying ${ }^{2}$ \\ ${ }^{1}$ Department of Clinical Laboratory, Central Hospital of Panzhihua City, Panzhihua, Sichuan 617067, P. R. China \\ ${ }^{2}$ Department of Laboratory Medicine, West China Hospital, Sichuan University, Chengdu, Sichuan 610041, P. R. China \\ *These authors have contributed equally to this work \\ Correspondence to: Binwu Ying, email: docbwy@126.com \\ Keywords: tuberculosis; LINC00152; genetic variants; susceptibility; adverse drug reaction \\ Received: June 15, $2017 \quad$ Accepted: December 03, $2017 \quad$ Published: December 14, 2017 \\ Copyright: Li et al. This is an open-access article distributed under the terms of the Creative Commons Attribution License 3.0 (CC \\ BY 3.0), which permits unrestricted use, distribution, and reproduction in any medium, provided the original author and source are \\ credited.
}

\section{ABSTRACT}

Recent studies indicate that the long intergenic non-coding RNA LINC00152 plays crucial roles in various human diseases. Here, we investigated whether levels of LINC00152 or its genetic variants correlate with the clinical features of tuberculosis (TB) in western Chinese patients. We genotyped the single nucleotide polymorphism LINC00152 rs80292941 in 476 TB patients and 475 healthy subjects using a customby-design 48-Plex SNPscan Kit, and measured relative levels of LINC00152 using RTqPCR. We observed that LINC00152 levels were lower in TB patients than controls. Moreover, rs80292941 TT genotype carriers had the lowest LINC00152 levels among TB patients, and rs80292941 AA genotype carriers are more likely to suffer from hepatotoxicity induced by antituberculosis therapy $[O R=3.97,95 \%=1.53-10.13, p$ $=0.002$ ]. Our findings strongly suggest that LINC00152 may promote TB progression and highlight rs80292941 single nucleotide polymorphism as a novel predisposition marker for antituberculosis drug-induced hepatotoxicity.

\section{INTRODUCTION}

Human tuberculosis (TB) resulting from Mycobacterium tuberculosis (MTB) infection remains a major public health threat all worldwide, with an estimated 10.4 million new cases and 1.7 million deaths occurring in 2016 [1]. TB control efforts face many major challenges, such as side effects from anti-TB chemotherapy, an increase in multidrug-resistant TB (MDR-TB) strains, and the prevalence of co-infection with human immunodeficiency virus (HIV) [2]. In spite of recent advances that have improved the diagnosis and treatment of TB, the molecular mechanisms underlying this disease remain unclear. There is thus a dire need to further investigate the specific molecules and signaling pathways involved in MTB infection and to develop novel molecular markers for the identification and treatment of human TB.

The Encyclopedia of DNA Elements (ENCODE) and Human Genome Project show that over $75 \%$ of the human genome is transcribed into primary transcripts, producing a broad range of non-coding RNA [3]. Long non-coding RNAs (lncRNAs) are RNA transcripts of more than 200 nucleotides that have a defective open reading frame [4] and participate in diverse biological processes [5, 6] underlying human disorders [7-9], especially malignant tumors. A newly delineated lncRNA, long intergenic noncoding RNA 00152 (LINC00152), is upregulated in human gastric tissues relative to adjacent cancer-free tissues [10] and might affect cell proliferation, cell cycle arrest, apoptosis, epithelial to mesenchymal transition (EMT), cell migration and invasion in gastric cancer [11]. Multiple studies support the oncogenic role of LINC00152 in several human carcinomas [12-15]. Taken together, these data suggest that IncRNA LINC00152 could serve as a new biomarker for the detection and treatment of multiple cancers. Deng et al [13] reported that hepatitis B virus (HBV) X-protein (HBx)-induced LINC00152 production could contribute to cancer progression in hepatocellular 
carcinoma associated with HBV. According to Wang et al [14], LINC00152 promotes renal cell carcinoma progression by acting as a microRNA-205 sponge and by epigenetically inhibiting P16 expression. Hu et al [12] showed that LINC00152 levels in peripheral circulation might be early predictors of esophageal squamous cell carcinoma (ESCC).

An increasing number of studies suggest that lncRNAs also promote human TB infection [16-19]. Wang et al [16] showed that lncRNA-CD244 suppresses IFN- $\gamma$ / TNF- $\alpha$ expression by mediating H3K27 trimethylation in infg/tnfa loci of CD8+ T cells. Another study [19] revealed distinct expression signatures for lncRNAs in macrophages after infection with H37Ra or H37Rv, showing that lncRNAs MIR3945HG V1 and MIR3945HG V1 could be used as diagnostic biomarkers for TB, with excellent area under the curve (AUC) values of 0.925 and 0.956 , respectively. However, the expression pattern, molecular function, and clinical relevance of LINC00152 in TB remains unexplored. Genetic association is the main approach used to identify whether genomic variant loci correlate with TB susceptibility. In addition to genetic variations within protein-coding genes, several single nucleotide polymorphisms (SNPs) located in non-coding genomic sequences have also been studied. We previously showed that rs12477677 within lncRNA AC0797767.4 is linked to a diminished predisposition to pulmonary tuberculosis (PTB) while potentially influencing clinical phenotypes of active TB [20]. Consequently, screening for SNPs from the large collection of human genomic protein non-coding regions is a promising strategy for the identification of TB susceptibility loci.

China is experiencing a serious TB epidemic. In western China, the annual incidence rate of PTB (up to 695 cases per 100,000 individuals) is remarkably higher than in other areas [21]. Therefore, whether LINC00152 and its genetic variations promote active $\mathrm{TB}$ is an attractive question deserving of further investigation. We thus carried out a case-controlled study of $476 \mathrm{~TB}$ cases and 475 healthy controls among western Chinese inhabitants to determine whether levels of LINC00152 and rs80292941 correlate with active TB disease risk and clinical features. Moreover, we performed a prospective follow-up analysis to investigate whether LINC00152 and its polymorphism rs80292941 affect the response of patients to anti-TB chemotherapy.

\section{RESULTS}

\section{Study subject demographic traits and clinical information}

Demographic and clinical features and quantitative inspection results of the cases and controls are shown in Table 1. We generally found no statistically significant differences in age or gender between the cases and controls, showing that both groups were frequencymatched with respect to age and sex. When comparing these laboratory parameters at baseline, TB patients showed distinct biochemical parameters and hematological blood patterns relative to those of healthy individuals, and cases with active TB presented elevated white blood cell and platelet counts as well as alanine transaminase (ALT) and aspartate transaminase (AST) levels compared to those of controls $(\mathrm{p}<0.001$ for all). TB patients exhibited lower hemoglobin $(\mathrm{Hb})$, hematocrit $(\mathrm{Hct})$, and erythrocyte levels ( $p<0.001$ for all) than healthy participants. As expected, the TB case group showed an obvious increase in C-reactive protein (CRP) and erythrocyte sedimentation rate (ESR) levels relative to those of the control group ( $p$ $<0.001$ for both).

As is shown in Table 1, we also reviewed a range of clinical and laboratory indexes correlated with TB disease, including clinical subtypes and conditions, etiological evidence, symptoms, and computed tomography (CT) results. We recruited 476 patients with active TB, of which 283 exhibited PTB (PTB, 59.45\%), 109 exhibited extrapulmonary tuberculosis (EPTB, 22.90\%), and 84 exhibited PTB combined with EPTB (PTB\&EPTB, 17.65\%). Among all cases, 46.64\% (222/476) had de novo TB, and $53.36 \%(254 / 476)$ had undergone anti-TB treatment for at least one month. In total, 55.99\% (173/309) of the TB-DNA positive rate was higher than positive rates for microscope smears and bacterial cultures [31.39\% (140/446), 19.83 (23/93), separately]. In our study, only a relatively small number $(n=93)$ of the patients with TB had undergone TB culture examination. Regarding clinical symptoms, patients (44.54\%) mainly complained of fever, and a majority of individuals (64.50\%) suffered from a cough. Of all of the 476 cases with TB, 360 subjects had received a CT scan on admission, of which $67.7 \%$ (244/360) had experienced bilateral lung damage, 28.33\% $(102 / 360)$ had developed a unilateral lung lesion, and only $3.89 \%$ (14/360) had not experienced lung injury.

For our case cohort, 272 TB patients received 2HREZ/4-7HRE [a regimen of isoniazid $(\mathrm{H})$, rifampicin $(\mathrm{R})$, ethambutol (E) and pyrazinamide (Z) for 2 months, followed by 4-7 months of HRE] while the remaining 204 received $2 \mathrm{HREZS} / 2-5 \mathrm{HRE}$ (S means streptomycin). All recruited TB patients underwent three routine laboratory tests at baseline before receiving anti-TB therapy. These patients were then subjected to monthly laboratory examination. Follow-up laboratory examination results for all of the patients are as follows: HB-valley: 116.85 \pm 25.02 g/L, PLT-valley: $234.75 \pm 114.32 \times 10^{9} / \mathrm{L}, \mathrm{WBC}-$ valley: $5.06(3.86-6.43) \times 10^{9} / \mathrm{L}$, ALT-peak: $28.50(15.25-$ 52.75), AST-peak: 29.00 (20.00-53.00), Creatinine-peak: 62.85 (50.00-77.00). Leukopenia (16.80\%, 80/476) was found to be the most common hematological adverse event involving RIF and INH use followed by anemia (15.33\%, 73/476) and drug-induced hepatotoxicity (15.33\%, $73 / 476)$. Only seven patients had suffered from chronic 
Table 1: Demographic/clinical characteristics of the study participants

\begin{tabular}{|c|c|c|c|}
\hline Features & TB $(n=476)$ & HC $(n=475)$ & $P$ \\
\hline \multicolumn{4}{|l|}{ General information } \\
\hline Age, mean $\pm \mathrm{SD}$ (years) & $41.47 \pm 19.29$ & $42.60 \pm 13.27$ & 0.291 \\
\hline Male/female & $289 / 187$ & $270 / 205$ & 0.225 \\
\hline \multicolumn{4}{|c|}{ Laboratory parameters before anti-TB } \\
\hline Erythrocyte $\left(\times 10^{12} / \mathrm{L}\right)$ & $4.20 \pm 0.82$ & $4.80 \pm 0.48$ & $<0.001$ \\
\hline Hematocrit (\%) & $37.00(32.00-41.00)$ & $45.00(40.00-48.00)$ & $<0.001$ \\
\hline Hemoglobin $(g / L)$ & $118.35 \pm 24.22$ & $145.59 \pm 15.16$ & $<0.001$ \\
\hline Platelets $\left(\times 10^{9} / \mathrm{L}\right)$ & $251.31 \pm 117.75$ & $170.92 \pm 46.89$ & $<0.001$ \\
\hline ALT (IU/L) & $22.00(16.00-32.00)$ & $11.00(7.00-16.75)$ & $<0.001$ \\
\hline AST (IU/L) & $18.00(14.00-23.00)$ & $23.00(19.00-29.00)$ & $<0.001$ \\
\hline $\mathrm{CRP}(\mathrm{mg} / \mathrm{L})$ & $19.85(6.04-67.13)$ & $5.49(1.70-18.42)$ & $<0.001$ \\
\hline $\operatorname{ESR}(\mathrm{mm} / \mathrm{h})$ & $48.00(22.00-78.50)$ & $20.38(8.43-56.61)$ & $<0.001$ \\
\hline \multicolumn{4}{|l|}{ TB clinical subtype n (\%) } \\
\hline PTB & $283(59.45)$ & & \\
\hline ЕРТВ & $109(22.90)$ & & \\
\hline РТВ \& ЕРТВ & $84(17.65)$ & & \\
\hline \multicolumn{4}{|l|}{ TB clinical condition n $(\%)$} \\
\hline De novo & $222(46.64)$ & & \\
\hline Retreated & $254(53.36)$ & & \\
\hline \multicolumn{4}{|c|}{ TB-related systemic symptoms n (\%) } \\
\hline Fever & $212(44.54)$ & & \\
\hline Loss weight & $149(31.30)$ & & \\
\hline Night sweat & $120(25.21)$ & & \\
\hline Poor appetite & $156(32.77)$ & & \\
\hline Fatigue & $99(20.80)$ & & \\
\hline \multicolumn{4}{|l|}{ Local chest symptoms n (\%) } \\
\hline cough & $307(64.50)$ & & \\
\hline hemoptysis & $72(15.12)$ & & \\
\hline Chest pain & $161(33.82)$ & & \\
\hline Dyspnea & $24(5.04)$ & & \\
\hline \multicolumn{4}{|l|}{ TB etiological evidence } \\
\hline TB-DNA positive/negative & 173/136 (55.99) & & \\
\hline Smear positive/negative & $140 / 446(31.39)$ & & \\
\hline Culture positive/negative & 23/93 (19.83) & & \\
\hline \multicolumn{4}{|l|}{ CT scan at baseline n (\%) } \\
\hline Bilateral lung damage & $244 / 360(67.78)$ & & \\
\hline Unilateral lung damage & $103 / 360(28.33)$ & & \\
\hline None lung damage & $14 / 360(3.89)$ & & \\
\hline
\end{tabular}




\begin{tabular}{|c|c|c|c|}
\hline Features & TB $(n=476)$ & $\mathrm{HC}(\mathrm{n}=475)$ & $P$ \\
\hline \multicolumn{4}{|c|}{ TB-related adverse drug reactions $n(\%)$} \\
\hline Anemia & $73(15.33)$ & & \\
\hline Leukopenia & $80(16.80)$ & & \\
\hline Thrombocytopenia & $31(6.51)$ & & \\
\hline Drug-induced hepatotoxicity & $73(15.33)$ & & \\
\hline Chronic kidney damage & $7(1.47)$ & & \\
\hline
\end{tabular}

Annotation: $\mathrm{TB}=$ tuberculosis; $\mathrm{HC}=$ healthy controls; $\mathrm{PTB}=$ pulmonary tuberculosis; $\mathrm{EPTB}=$ extra-pulmonary tuberculosis; PTB \& EPTB = pulmonary tuberculosis combined with extra-pulmonary tuberculosis.

kidney damage (CKD) associated with anti-tuberculosis drugs (ATDs).

\section{SNP rs80292941 association with TB susceptibility}

\section{Genotyping results}

The candidate SNP rs80292941 within the LINC00152 gene sequence was genotyped successfully in all 476 patients with TB disease and in the 475 control individuals. Genotype distributions of polymorphism rs80292941 in the control group were in compliance with Hardy-Weinberg equilibrium $(\mathrm{HWE})(\mathrm{p}$-value $=0.658)$, and the minor allele frequency (MAF) was 0.114.

\section{Candidate rs80292941 association analysis}

Allele and genotypic distributions of the rs 80292941 locus between the TB cases and healthy populations are shown in Table 2. Proportions of minor allele $\mathrm{T}$ in rs80292941 loci in the case and control groups were $11.13 \%$ and $11.68 \%$, respectively. The allelic distribution of this locus was largely analogous between the two groups with a p-value of $0.706(\mathrm{OR}=0.95,95 \% \mathrm{CI}=0.71-1.26)$. For the cases with active TB, the frequencies of genotypes TT, AT and AA were $2.10 \%, 18.07 \%$, and $77.68 \%$, respectively, and TT, AT and AA genotype rates of the controls were $1.06 \%, 21.26 \%$, and $77.68 \%$, respectively, with a statistically significant p-value of 0.220 . To further investigate genotype distribution differences between these 2 groups, we performed a genetic model analysis (including a dominant and recessive model). Our analyses failed to find any statistically significant differences in dominant and recessive genetic patterns between the cases and controls ( $p>0.05$ for all as shown in Table 2).

As several given SNPs may be correlated with the specific TB subtype reported in previous studies [20], we conducted multiple subgroup analyses. We classified all TB cases into three subgroups based on TB clinical subtypes: 283 PTB patients, 109 EPTB patients, and 84 patients with PTB\&EPTB. According to the TB clinical treatment condition, all cases were stratified as two subsets: 222 newly diagnosed TB cases and 254 retreated cases. Unfortunately, we did not find any positive association between these TB subsets and healthy controls (data not shown).

\section{Relationship between rs80299241 genotypes and TB phenotypes}

We comprehensively assessed the potential correlation between different rs80292941 genotypes and TB disease manifestation. Several common indexes of active TB disorder were evaluated (i.e., TB-related systemic and local symptoms, complete blood cell test results, TB etiological analysis results, ESR, CRP levels, and lung lesions observed from chest CT scans). Due to infrequent proportions of minor homozygous genotypes (TT) found in rs80292941, we drew comparisons based on the dominant pattern found (AA vs. AT+TT). For the rs80292941 locus, none of the analyzed variations correlated with genotype distributions (Table 3 ). We only found that patients with the minor allele (T allele) and with both homozygotes and heterozygotes appeared to suffer less from night sweats than those with the homozygous major allele (AA), although the related p-value was not statistically significant $(\mathrm{p}=0.064$, Table 3$)$.

\section{Comprehensive analysis of changes in LINC00152 expression levels in TB cases}

\section{Expressive levels of LINC00152 between cases and controls}

Our real-time PCR quantitative results showed that the case group had lower LINC00152 relative levels normalized to the experimental endogenous control, compared to those of the control group [cases: 7.45 (3.6015.51); controls: 16.77 (6.81-24.05), $\mathrm{p}<0.001]$ (Table 4, Figure 1), implying that LINC00152 might modulate the development and progression of TB. 
Table 2: SNP rs80292941 in relation to susceptibility to TB

\begin{tabular}{|c|c|c|c|c|}
\hline SNP variations & Case (n \%) & Control (n \%) & $P$ & $95 \% \mathrm{CI}$ \\
\hline \multicolumn{5}{|l|}{ Allele } \\
\hline $\mathrm{T}$ & $106(11.13)$ & $111(11.68)$ & 0.706 & $0.95(0.71-1.26)$ \\
\hline A & 846 (88.87) & $839(88.32)$ & & \\
\hline \multicolumn{5}{|l|}{ Genotype } \\
\hline TT & $10(2.10)$ & $5(1.06)$ & 0.220 & \\
\hline AT & $86(18.07)$ & $101(21.26)$ & & \\
\hline $\mathrm{AA}$ & $380(79.83)$ & $369(77.68)$ & & \\
\hline \multicolumn{5}{|l|}{ Dominant model } \\
\hline $\mathrm{TT}+\mathrm{AT}$ & $96(20.17)$ & $106(22.32)$ & 0.418 & $0.88(0.64-1.20)$ \\
\hline AA & $380(79.83)$ & $369(77.68)$ & & \\
\hline \multicolumn{5}{|l|}{ Recessive model } \\
\hline TT & $10(2.10)$ & $5(1.05)$ & 0.195 & $2.01(0.68-5.95)$ \\
\hline $\mathrm{AT}+\mathrm{AA}$ & $466(97.90)$ & $470(9.95)$ & & \\
\hline
\end{tabular}

Annotation: $p$-value was calculated by Chi-square test.

\section{Investigation of the role of SNP rs80292941 in LINC00152 expression}

\footnotetext{
Using lncRNA SNP bioinformatics software [22] (http://bioinfo.life.hust.edu.cn/lncRNASNP/), we found that candidate genetic variation rs80292941 might influence the binding of lncRNA LINC00152 to several microRNAs (miRNAs) including both gains and losses. We hypothesized that rs80292941 is indirectly associated with LINC00152 expression. To test this, we evaluated the effects of rs80292941 on LINC00152 levels. Relative levels of LINC00152 derived from all 476 TB cases and 475 controls were compared across the three different SNP genotypes. We found substantial discrepancies in levels of LINC00152 among different rs80292941 genotypes for the case groups [TT: 2.79 (1.86-6.29) versus. AT: 9.38 (3.99-21.01) versus. AA: 7.45 (3.75-15.22), $\mathrm{p}=0.018$, Table 5], among which homozygous minor allele (TT) carriers expressed the lowest LINC00152 transcript levels. The LINC00152 levels of three different genotypes were comparable for the healthy control group (TT: 15.75 (5.45-24.98) versus. AT: 17.36 (6.54-24.56) versus. AA: 16.77 (6.81$24.05), \mathrm{p}=0.973$, Table 5). For cases involving TB infection, we surprisingly found that LINC00152 levels had remarkably decreased in all three rs80292941 genotypes relative to those of healthy subjects ( $\mathrm{p}=$ 0.040 for TT, $\mathrm{p}=0.008$ for AT, $\mathrm{p}<0.001$ for AA as shown in Table 5). Interestingly, Table 5 shows that TB patients with TT genotypes may show the greatest decline in LINC00152 levels relative to those of the other two genotypes.
}

\section{LINC00152 and rs80292941 associations with adverse drug reactions related to $\mathrm{TB}$ treatment}

It was previously reported that upregulated LINC00152 is positively associated with resistance to oxaliplatin chemotherapy in colon cancer [23]. In this study, we innovatively assessed whether the LINC00152 and its polymorphism rs80292941 correlated with adverse drug reactions induced by TB chemotherapy. We analyzed common TB-related adverse reactions in our datasets involving cases of anemia, leukopenia, thrombocytopenia, hepatotoxicity, and CKD, which were dependent on positive laboratory examination results and clinical manifestations when possible. We failed to observe any associations between LINC00152 relative levels and the occurrence of five adverse drug responses from TB chemotherapy (Table 6). However, the TB non-susceptibility locus, the dominant model of rs80292941 [AA/(AT+TT)], is closely associated with the presence of drug-induced hepatotoxicity in the dominant model. Patients carrying homozygous for the A allele (AA genotype) were more likely to bear hepatotoxicity resulting from anti-TB therapy than $\mathrm{T}$ allele carriers (AA: $17.89 \%$ vs. AT + TT: $5.21 \%)$ with an estimated $\mathrm{p}=0.002$ $(\mathrm{OR}=3.97,95 \% \mathrm{CI}=1.53-10.13$ as shown in Table 7$)$.

\section{DISCUSSION}

Long intergenic non-coding RNA 00152, LINC00152, is situated in chromosome 2p11.2 and has a transcript with 828 nucleotides. Here, we explored whether 
Table 3: Correlation between rs80292941 polymorphism and TB phenotype traits

\begin{tabular}{|c|c|c|c|}
\hline Characterizations & $\mathrm{AA}(\mathrm{N}=\mathbf{3 8 0})$ & $\mathrm{AT}+\mathrm{TT}(\mathrm{N}=96)$ & $P$ \\
\hline \multicolumn{4}{|l|}{ Clinical phenotypes n (\%) } \\
\hline \multicolumn{4}{|l|}{ Systemic symptoms } \\
\hline Fever & $170(80.2)$ & $42(43.75)$ & 0.862 \\
\hline Weight loss & $128(40.76)$ & $32(33.33)$ & 0.948 \\
\hline Night sweat & $111(32.48)$ & $19(19.79)$ & 0.064 \\
\hline Poor appetite & $136(38.22)$ & $30(31.25)$ & 0.404 \\
\hline Fatigue & $92(38.22)$ & $18(18.75)$ & 0.257 \\
\hline \multicolumn{4}{|l|}{ Local chest symptoms } \\
\hline cough & $242(57.76)$ & $65(67.71)$ & 0.462 \\
\hline hemoptysis & $59(14.29)$ & $13(13.54)$ & 0.628 \\
\hline chest pain & $68(34.16)$ & $17(17.71)$ & 0.966 \\
\hline dyspnea & $20(7.45)$ & $4(4.17)$ & 0.859 \\
\hline \multicolumn{4}{|l|}{ Laboratory traits } \\
\hline \multicolumn{4}{|l|}{ Full blood cell counts } \\
\hline Erythrocyte $\left(\times 10^{12} / \mathrm{L}\right)$ & $4.18 \pm 0.84$ & $4.28 \pm 0.76$ & 0.295 \\
\hline Hematocrit (\%) & $37.00(32.00-41.00)$ & $38.00(32.00-42.00)$ & 0.471 \\
\hline Hemoglobin $(g / L)$ & $118.10 \pm 24.40$ & $119.36 \pm 23.56$ & 0.647 \\
\hline Platelets $\left(\times 10^{9} / \mathrm{L}\right)$ & $252.18 \pm 118.75$ & $247.86 \pm 114.22$ & 0.749 \\
\hline Leucocytes $\left(\times 10^{9} / \mathrm{L}\right)$ & $6.49(4.80-8.90)$ & $6.29(4.73-8.63)$ & 0.362 \\
\hline Neutrophils $\left(\times 10^{9} / \mathrm{L}\right)$ & $4.64(3.15-6.58)$ & $4.19(3.01-6.26)$ & 0.172 \\
\hline Lymphocytes $\left(\times 10^{9} / \mathrm{L}\right)$ & $1.07(0.75-1.52)$ & $1.25(0.79-1.71)$ & 0.152 \\
\hline Monocytes $\left(\times 10^{9} / \mathrm{L}\right)$ & $0.46(0.31-0.67)$ & $0.45(0.29-0.62)$ & 0.407 \\
\hline \multicolumn{4}{|l|}{ Routine TB markers } \\
\hline $\mathrm{CRP}(\mathrm{mg} / \mathrm{L})$ & $22.35(7.63-76.85)$ & $19.35(6.81-69.25)$ & 0.214 \\
\hline $\operatorname{ESR}(\mathrm{mm} / \mathrm{h})$ & $45.00(23.00-73.00)$ & $43.00(22.00-74.00)$ & 0.824 \\
\hline \multicolumn{4}{|l|}{ TB etiological assays } \\
\hline Positive TB-DNA & $136(55.28)$ & $37(58.73)$ & 0.623 \\
\hline Positive smear & $111(31.18)$ & $29(32.22)$ & 0.849 \\
\hline \multicolumn{4}{|l|}{ CT scanning } \\
\hline Bilateral lung lesion & $191(66.55)$ & $55(75.34)$ & 0.204 \\
\hline Unilateral lung lesion & $86(29.97)$ & $17(23.29)$ & \\
\hline
\end{tabular}

Table 4: Relative expression level of $L I N C 00152$ in cases and controls

\begin{tabular}{lccc}
\hline LINC00152 & TB $(\mathbf{n}=\mathbf{4 7 6})$ & HC $(\mathbf{n}=\mathbf{4 7 5})$ & $\boldsymbol{P}$ \\
\hline Relative level $\left(\times 10^{4}\right)$ & $7.45(3.60-15.51)$ & $16.77(6.81-24.05)$ & $<\mathbf{0 . 0 0 1}$ \\
\hline
\end{tabular}

Annotation: The data was displayed as median (interquartile range, IQR) and was compared with Mann-Whitney U method. 
expression of LINC00152 and its variant rs80292941 correlates with TB predisposition, disease presentation, or adverse reactions to anti-TB drugs. We found that LINC00152 expression is markedly decreased in cases of TB infection and that different rs80292941 genotypes modulate LINC00152 expression. Furthermore, we found that the rs80292941 locus correlates with increased hepatotoxicity levels after anti-TB therapy.

In our study, polymorphism rs80292941 in the LINC00152 gene did not increase susceptibility to TB in western Chinese. However, for patients with TB, we found significant differences in LINC00152 rs80292941 levels for three genotype groups (TT vs. AT vs. AA genotype), with homozygous TT genotype carriers showing the lowest levels. In addition, patients with the TT genotype may contribute most to the downregulation of LINC00152 for the case group when compared to the healthy controls. SNP rs80292941 located in the untranslated region of the LINC00152 gene may affect the interaction between LINC00152 and many miRNAs and may subsequently dysregulate LINC00152 transcript expression.
Supplementary Figure 1 shows the miRNA binding locus within rs 80292941 in the presence of the T allele. On the other hand, in the presence of the A allele, the binding locus is lost (Supplementary Figure 2). MiRNA is involved in the function of RNA-induced silencing complexes, which play a role in degrading complimentary target mRNAs or blocking their translation. As for noncoding RNA, Zhang et al. demonstrated that miR-376c-3p negatively regulated the expression of LINC00152 in colorectal cancer cells, restricting cell viability and stimulating cell apoptosis [24]. Therefore, here we hypothesize that some functional miRNAs might bind rs80292941 TT, thereby lowering LINC00152 expression, which should be further tested with functional assays.

We found LINC00152 to be substantially downregulated in TB cases relative to controls. Compelling data from previous studies have generally shown that lncRNA LINC00152 is upregulated in many types of human malignant cancers (including gastric cancer, colorectal cancer, etc.) and accelerates carcinoma progression and migration through diverse molecular

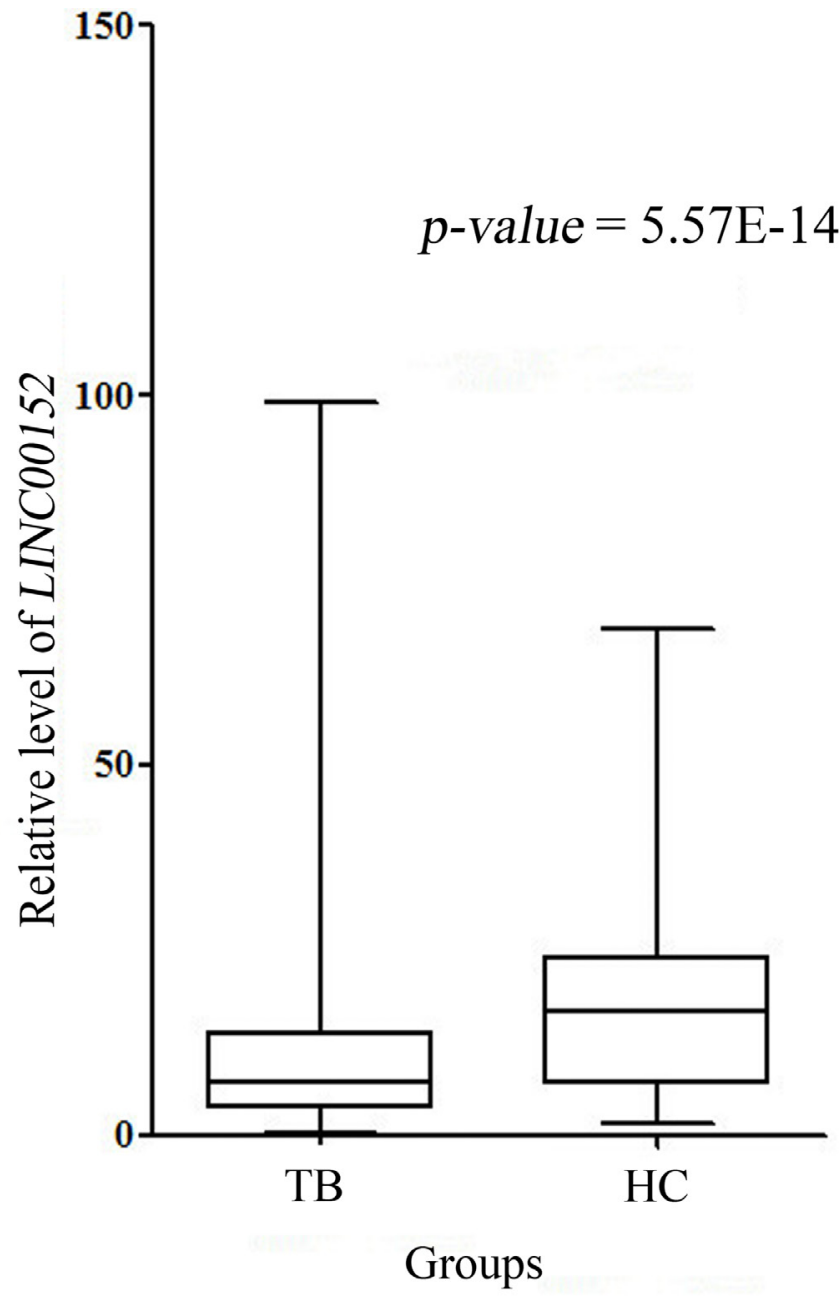

Figure 1: LINC00152 relative expression in cases and controls.

Note: There was abundant reduction in LINC00152 expression in cases compared with controls $(\mathrm{p}<0.001)$. 
Table 5: Relative expression level of LINC00152 in different rs0292941 genotypes between cases and controls

\begin{tabular}{lccc}
\hline Genotypes & Cases & Controls & $\begin{array}{c}P \text { between cases and } \\
\text { controls }\end{array}$ \\
\hline TT genotype & $2.79(1.86-6.29)$ & $15.75(5.45-24.98)$ & $\mathbf{0 . 0 4 0}$ \\
AT genotype & $9.38(3.99-21.01)$ & $17.36(6.54-24.56)$ & $\mathbf{0 . 0 0 8}$ \\
AA genotype & $7.45(3.75-15.22)$ & $16.77(6.81-24.05)$ & $<\mathbf{0 . 0 0 1}$ \\
\hline
\end{tabular}

Annotation: $p=0.018$ and 0.973 for three genotypes in case group and control group, respectively.

Table 6: Relationships between LINC00152 transcript and TB-related drug adverse responses

\begin{tabular}{|c|c|c|}
\hline Drug adverse reactions & LINC00152 levels & $\boldsymbol{P}$ \\
\hline Anemia n (\%) & & 0.348 \\
\hline Presence $(73,15.33)$ & $9.63(3.52-13.72)$ & \\
\hline Absence $(403,84.67)$ & $7.56(3.69-17.63)$ & \\
\hline Leukopenia n (\%) & & 0.769 \\
\hline Presence $(80,16.80)$ & $7.20(3.91-14.80)$ & \\
\hline Absence $(396,83.20)$ & $7.56(3.55-15.97)$ & \\
\hline Thrombocytopenia n (\%) & & 0.775 \\
\hline Presence $(31,6.51)$ & $6.72(2.87-30.01)$ & \\
\hline Absence $(445,93.49)$ & $7.56(3.73-15.22)$ & \\
\hline Drug-induced hepatotoxicity n (\%) & & 0.602 \\
\hline Presence $(73,15.33)$ & $6.35(3.56-15.06)$ & \\
\hline Absence $(403,84.67)$ & $7.61(3.65-15.70)$ & \\
\hline Chronic kidney damage n (\%) & & 0.419 \\
\hline Presence $(7,1.47)$ & $5.40(3.45-12.42)$ & \\
\hline Absence $(469,98.53)$ & $7.50(3.60-15.81)$ & \\
\hline
\end{tabular}

Table 7: Relationships between rs80292941 and TB-related drug adverse responses in the dominant model

\begin{tabular}{|c|c|c|c|c|}
\hline Drug adverse reactions & $\mathrm{AA}(\mathrm{N}=\mathbf{3 8 0})$ & $\mathrm{AT}+\mathrm{TT}(\mathrm{N}=96)$ & $P$ & OR $(95 \% \mathrm{CI})$ \\
\hline Anemia n (\%) & $57(15.00)$ & $16(16.67)$ & 0.686 & $0.88(0.48-1.62)$ \\
\hline Leukopenia n (\%) & $62(16.32)$ & $18(18.75)$ & 0.569 & $0.84(0.47-1.51)$ \\
\hline Thrombocytopenia n (\%) & $25(6.58)$ & $6(6.25)$ & 0.907 & $1.06(0.42-2.65)$ \\
\hline $\begin{array}{l}\text { Drug-induced } \\
\text { hepatotoxicity n (\%) }\end{array}$ & $68(17.89)$ & $5(5.21)$ & 0.002 & $3.97(1.53-10.13)$ \\
\hline $\begin{array}{l}\text { Chronic kidney damage } n \\
(\%)\end{array}$ & $4(1.05)$ & $3(3.13)$ & 0.149 & $0.33(0.73-1.50)$ \\
\hline
\end{tabular}


mechanisms. For example, Cai et al [25] showed that LINC00152 overexpression induced by transcription factor specificity protein 1 (SP1) might be involved in the phosphatidylinositol 3-kinase (PI3K)/AKT pathway and act as an oncogene in gallbladder cancer (GBC). Further mechanistic analyses also suggested that LINC00152 acts as a competing endogenous RNA (ceRNA) to sponge miR-138 and subsequently inhibit HIF-1 $\alpha$ (hypoxia inducible factor- $1 \alpha$ ) expression, ultimately contributing to GBC metastasis and epithelial-mesenchymal transition [26]. Recently, more attention has been dedicated to how lncRNAs functioning as ceRNAs are involved in biological development and human disorders. We speculate that LINC00152 might promote TB infection by interacting with miRNAs markers. From the lncRNA SNP database, we identified 38 miRNAs that might target LINC00152 molecules as shown in Figure 2. Of these miRNAs, several miRNA molecules have been reported to promote TB infection development and progression. The most interesting ones seem to be the following four candidate miRNAs: miR-16 [27], miR-125b [28], miR-206 [29], and miR-424 [30]. Indeed, the published literature shows that levels of these four candidate miRNAs are increased in TB patient samples and MTB-infected cells. Our experimental results, bioinformatics predictions, and published articles suggest that LINC00152 acting as an miRNA sponge might interact with these candidate miRNAs to promote TB development and progression.

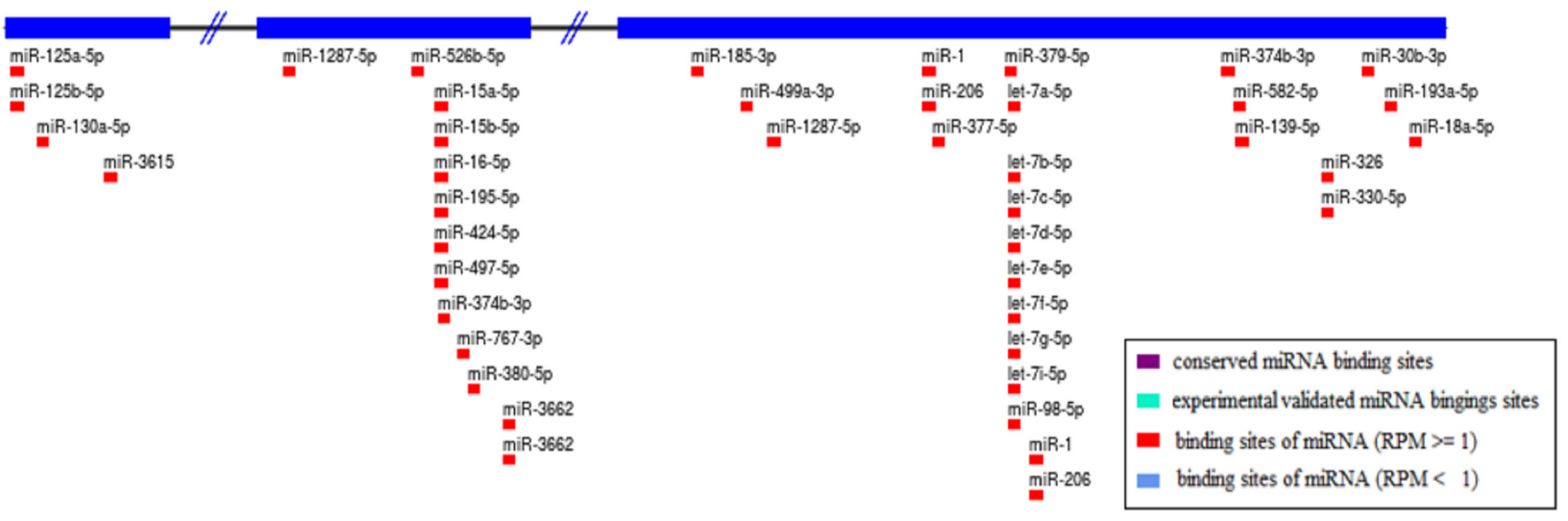

Figure 2: Prediction of miRNAs targeting LINC00152.

Note: A total of 38 kinds of miRNAs were obtained through the lncRNA SNP database.

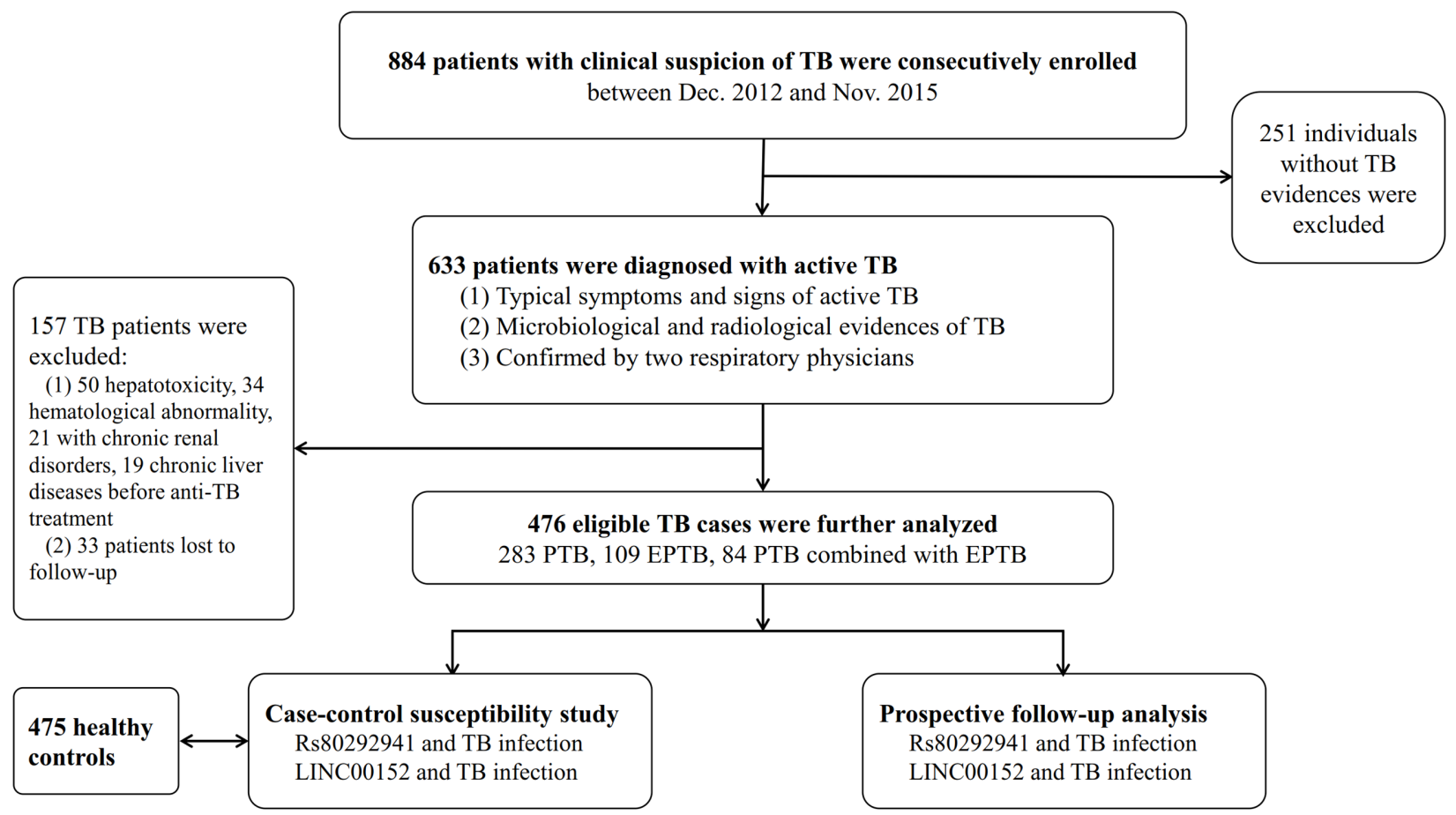

Figure 3: Flow diagram of enrollment of study participants. 
Our observations can thus guide future studies on the biological mechanisms of LINC00152 in TB infection.

The management of TB disease is frequently associated with serious complications, including several adverse reactions to ATDs. Liver injury is the most common and serious adverse effect of TB therapy involving isoniazid, rifampicin, and pyrazinamide, which affects 5\%-28\% of TB patients [31-32]. A better understanding of risk factors of anti-TB induced liver injury might help prevent this condition, which occasionally contributes to the discontinuation of treatment. Some studies have reported several genetic risk biomarkers such as rs1695 in the GSTP1 gene [32$33]$ and the slow-acetylator genotype of the NAT2 gene $[32,34]$. Other studies have mainly focused on drug metabolizing enzyme gene variants as candidate markers of drug-induced liver injury. We are the first to find that LINC00152 rs80292941 may be a TB predisposition factor and that AA genotype carriers are almost four times more likely to develop RIP- and INH-based antiTB liver injuries than patients with AT/TT genotypes. Yue et al reported that LINC00152 is involved in xaliplatin (L-OHP) drug resistance in cases of colon carcinoma [23]. These results indicate that LINC00152 and its variant may be involved in host responses to drug treatment. However, their roles in adverse drug reactions remain unclear, warranting further investigation.

Although our study successfully highlights the significance of LINC00152 expression and of its polymorphism rs80292941 in susceptibility, clinical disease features, and adverse responses to TB drugs, our study suffers from several limitations. First, only one SNP within LINC00152 among western Chinese was studied. Future studies should explore more variants in genetically diverse populations. Second, future mechanistic investigations must elucidate specific biological roles of LINC00152 in TB infection. Third, higher risks of rs80292941 AA genotyping associated with anti-TB drugrelated liver injury require further investigation through multi-center studies and functional experimental research.

In conclusion, we report that LINC00152 expression is significantly downregulated in TB cases and that LINC00152 rs80292941 may serve as a predisposing factor for anti-TB induced hepatotoxicity. Our findings strongly suggest that LINC00152 and its variant promote TB infection and progression.

\section{MATERIALS AND METHODS}

\section{Study subjects}

In our prospective observational study, we preliminarily enrolled 884 patients and 475 unrelated healthy controls from the West China Hospital of Sichuan University in western China from Dec. 2012 to Nov. 2015. TB diagnoses were made for 633 patients based on typical symptoms of active TB disease and based on the identification of microbiological and radiological evidence of active TB. All cases of active TB were confirmed by two experienced respiratory physicians. Regimens with antituberculosis drugs (ATDs) were administered for four or more months, according to the clinical practices recommended by the World Health Organization (WHO) [35]. Patients meeting the following criteria were excluded: (a) no microbiological or radiological evidence of TB; (b) signs of hepatotoxicity, renal injury, and hematological toxicity prior to anti-TB chemotherapy; (c) positive serological testing for $\mathrm{HIV}, \mathrm{HBV}$, or $\mathrm{HCV}$; (d) history of chronic liver/kidney/hematological system disease; (e) pregnancy; (f) poor adherence to treatment or to one-year follow-up observations. Controls were recruited from healthy blood donors who had negative interferon gamma release assay (IGRA) results, normal physical examination results, and no history of TB.

Demographic data and clinical information of the studied population were reviewed from medical records. Ethylenediaminetetraacetic acid (EDTA)-anticoagulated whole blood samples were collected from each subject for genotyping and lncRNA expression. After starting ATDs, biochemical parameters, hematological blood counts and urinalyses were measured monthly to evaluate adverse reactions to ATDs until the end of the treatment period. Adverse reactions to ATDs covered cases of drug-induced hepatotoxicity, CKD, and hematological toxicity. Druginduced hepatotoxicity was determined according to criteria on drug-induced liver disorders [36]. Individuals presenting AST and ALT levels of more than twice the maximum limit were considered to have hepatotoxicity. CKD was evaluated as the consensus of drug-induced kidney disease by Mehta et al, defined as the persistence of kidney injury (hematuria, proteinuria, casts, and so on) for $>90$ days [37]. Anemia $\left(\mathrm{Hb} \leq 90 \times 10^{12} / \mathrm{L}\right)$, leukopenia (absolute counts $<3.5 \times 10^{9} / \mathrm{L}$ ), and thrombocytopenia (absolute counts $<90 \times 10^{9} / \mathrm{L}$ ) were considered forms of hematological toxicity [38]. Figure 3 shows a flow diagram of the process used to enroll participants. This hospital-based case-control study was approved of by the Review Board of West China Hospital, Sichuan University (Sichuan, China). All individuals provided written informed consent to participate in the study.

\section{Single nucleotide polymorphism selection and genotyping}

Rs80292941 of LINC00152 was obtained from the dbSNP (http://www.ncbi.nlm.nih.gov/projects/SNP/.) and lncRNA SNP databases (http://bioinfo.life.hust.edu. $\mathrm{cn} / \mathrm{lncRNASNP} /$ ). It is located at position $87,480,257$ in chromosome 2 (GRCh38.p7). Genomic DNA was extracted from blood samples using a QIAamp ${ }^{\circledR}$ DNA blood mini kit (Qiagen, Germany). DNA samples (10 ng) were amplified and genotyped using a custom-by- 
design 48-Plex SNPscan Kit (Genesky Biotechnologies Inc., Shanghai, China) as described above according to the manufacturer's recommendations. The commercial kit is based on patented SNP genotyping technology with a double-ligation and multiplex fluorescence PCR developed by Genesky Biotechnologies Inc. For quality control purposes, repeated analyses were carried out for $10 \%$ of the randomly selected samples presenting high DNA quality.

\section{Measurement of $L I N C 00152$ expression}

Total RNA was isolated from the peripheral blood mononuclear cells of patients using TRIzol reagent (Invitrogen, CA, USA) and was then converted into complementary DNA (cDNA) using an Omniscript ${ }^{\circledR}$ Reverse Transcription Kit (Qiagen, CA, USA). The RT-qPCR reaction involved $5 \mu \mathrm{l}$ of SYBR $^{\circledR}$ Premix Ex Taq ${ }^{\mathrm{TM}}$ II (Takara, Dalian, China), $1 \mu \mathrm{l}$ of $10 \mu \mathrm{M}$ forward and reverse primer (forward: 5'-GCTCCTGGCACAGTCTTTTCTC-3', reverse: 5'-GGCTGGCAAGTTTCCAATATACA-3'), $3 \mu$ l of water and $1 \mu \mathrm{l}$ of cDNA template. RT-qPCR was performed in a LightCycler ${ }^{\circledR} 480$ Real-Time PCR System (Roche Diagnostics, NJ, USA) involving an initial denaturation of $15 \mathrm{~min}$ at $96^{\circ} \mathrm{C}$, amplification for 45 cycles by denaturing at $96^{\circ} \mathrm{C}$ for $10 \mathrm{~s}$, annealing at $60^{\circ} \mathrm{C}$ for $30 \mathrm{~s}$ and extension at $72^{\circ} \mathrm{C}$ for $30 \mathrm{~s}$. The samples were denatured at $95^{\circ} \mathrm{C}$ for $30 \mathrm{~s}$ and then heated to $60^{\circ} \mathrm{C}$ for $30 \mathrm{~s}$ at a rate of $0.1^{\circ} \mathrm{C} / \mathrm{s}$. Fluorescence was measured to generate the melting curve of the amplified products. Raw data were analyzed using Gene Scanning v1.2 software. LINC00152 expression was normalized to the endogenous control GAPDH and was calculated according to the $2-\Delta \mathrm{CT}$ method.

\section{Statistical analysis}

Chi-square and Mann-Whitney U test results were evaluated for categorical and continuous variables. HWE among the controls was determined through Fisher's exact test. Associations between rs80292941 and LINC00152 expression, TB risk, and adverse drug reactions were evaluated on the basis of allele frequency distributions/ genetic models (additive, dominant and recessive model) and were calculated through a Chi-square test. The strength of association was estimated with odds ratios (ORs) and at a 95\% confidence interval (CI). The above listed statistical methods were performed using SPSS version 20.0 (IBM, Chicago, USA). All tests were twosided, and statistical significance was set at an alpha level of 0.05 .

\section{Abbreviations}

ALT: alanine transaminase; AST: aspartate transaminase; ATDs: antituberculosis drugs; ceRNA: competing endogenous RNA; CI: confidence interval;
CKD: chronic kidney damage; CRP: C-reactive protein; CT: computed tomography; EMT: epithelial to mesenchymal transition; ENCODE: Encyclopedia of DNA Elements; EPTB: extra-pulmonary tuberculosis; ESCC: early occurrence of esophageal squamous cell carcinoma; ESR: erythrocyte sedimentation rate; GBC: gallbladder cancer; $\mathrm{Hb}$ : hemoglobin; $\mathrm{HBV}$ : hepatitis $\mathrm{B}$ virus; $\mathrm{HC}$ : healthy controls; Hct: hematocrit; HIF-1 $\alpha$ : hypoxia inducible factor-1 $\alpha$; HIV: human immunodeficiency virus; HWE: Hardy-Weinberg equilibrium; IGRA: interferon gamma release assay; INH: isoniazid; LINC00152: long intergenic non-coding RNA 00152; LncRNA: long noncoding RNA; MAF: the minor allele frequency; MDR-TB: multidrug-resistant tuberculosis; MTB: Mycobacterium tuberculosis; ORs: odds ratios; PI3K: phosphatidylinositol 3-kinase; PTB \& EPTB: pulmonary tuberculosis combined with extra-pulmonary tuberculosis; PTB: pulmonary tuberculosis; RIF: rifampicin; SNP: single nucleotide polymorphism; SP1: specificity protein 1; TB: tuberculosis; WHO: World Health Organization.

\section{Author contributions}

J.L. and L.J.W. wrote the main manuscript text and participated in the experiments all the way. X.J.H. and Z.Z.Z. participated in modifying the manuscript; W.H.G. and J.L.C. participated in the analysis of data and prepared Tables and figures; W.H.G., J.L.C. and M.J.W. engaged in the acquisition of data (laboratory or clinical); B.W.Y. and X.J.H. designed the study. All authors have reviewed the manuscript.

\section{ACKNOWLEDGMENTS}

The authors are grateful to Shanghai Genesky BioTech Genetic Core Lab for providing assistance with genotyping.

\section{CONFLICTS OF INTEREST}

The authors declare no competing financial interests.

\section{FUNDING}

This work was supported by grants from the National Natural Science Foundation of China [81472026, 81672095] and the Projects in the Science and Technology Department of Sichuan Province pillar program [2014SZ0208].

\section{REFERENCES}

1. World Health Organization. Global tuberculosis report 2017. Available from: http://www.who.int/tb/publications/ global_report/en/ (Date of access: 10/20/2017) (2017). 
2. Schnippel K, Firnhaber C, Berhanu R, Page-Shipp L, Sinanovic E. Adverse drug reactions during drug-resistant TB treatment in high HIV prevalence settings: a systematic review and meta-analysis. J Antimicrob Chemother. 2017. http://doi.org/10.1093/jac/dkx107.

3. Djebali S, Davis CA, Merkel A, Dobin A, Lassmann T, Mortazavi A, Tanzer A, Lagarde J, Lin W, Schlesinger F, Xue C, Marinov GK, Khatun J, et al. Landscape of transcription in human cells. Nature. 2012; 489:101-8. http://doi.org/10.1038/nature11233.

4. Hung T, Chang HY. Long noncoding RNA in genome regulation: prospects and mechanisms. RNA Biol. 2010; 7:582-5.

5. Wang KC, Chang HY. Molecular mechanisms of long noncoding RNAs. Mol Cell. 2011; 43:904-14. http://doi. org/10.1016/j.molcel.2011.08.018.

6. Carpenter S, Aiello D, Atianand MK, Ricci EP, Gandhi P, Hall LL, Byron M, Monks B, Henry-Bezy M, Lawrence JB, O’Neill LA, Moore MJ, Caffrey DR, et al. A long noncoding RNA mediates both activation and repression of immune response genes. Science. 2013; 341:789-92. http:// doi.org/10.1126/science.1240925.

7. Bartonicek N, Maag JL, Dinger ME. Long noncoding RNAs in cancer: mechanisms of action and technological advancements. Mol Cancer. 2016; 15:43. http://doi. org/10.1186/s12943-016-0530-6.

8. Mirza AH, Kaur S, Brorsson CA, Pociot F. Effects of GWAS-associated genetic variants on lncRNAs within IBD and T1D candidate loci. PLoS One. 2014; 9:e105723. http:// doi.org/10.1371/journal.pone.0105723.

9. Lorenzen JM, Thum T. Long noncoding RNAs in kidney and cardiovascular diseases. Nat Rev Nephrol. 2016; 12:360-73. http://doi.org/10.1038/nrneph.2016.51.

10. Cao WJ, Wu HL, He BS, Zhang YS, Zhang ZY. Analysis of long non-coding RNA expression profiles in gastric cancer. World J Gastroenterol. 2013; 19:3658-64. http://doi. org/10.3748/wjg.v19.i23.3658.

11. Zhao J, Liu Y, Zhang W, Zhou Z, Wu J, Cui P, Zhang Y, Huang G. Long non-coding RNA LINC00152 is involved in cell cycle arrest, apoptosis, epithelial to mesenchymal transition, cell migration and invasion in gastric cancer. Cell Cycle. 2015; 14:3112-23. http://doi.org/10.1080/15384101 .2015 .1078034 .

12. Hu HB, Jie HY, Zheng XX. Three circulating lncRNA predict early progress of esophageal squamous cell carcinoma. Cell Physiol Biochem. 2016; 40:117-25. http:// doi.org/10.1159/000452529.

13. Deng X, Zhao XF, Liang XQ, Chen R, Pan YF, Liang J. LINC00152 promotes cancer progression in hepatitis B virus-associated hepatocellular carcinoma. Biomed Pharmacother. 2017; 90:100-8. http://doi.org/10.1016/j. biopha.2017.03.031.

14. Wang Y, Liu J, Bai H, Dang Y, Lv P, Wu S. Long intergenic non-coding RNA 00152 promotes renal cell carcinoma progression by epigenetically suppressing P16 and negatively regulates miR-205. Am J Cancer Res. 2017; 7:312-22.

15. Yu X, Lin Y, Sui W, Zou Y, Lv Z. Analysis of distinct long noncoding RNA transcriptional fingerprints in pancreatic ductal adenocarcinoma. Cancer Med. 2017; 6:673-80. http://doi.org/10.1002/cam4.1027.

16. Wang Y, Zhong H, Xie X, Chen CY, Huang D, Shen L, Zhang H, Chen ZW, Zeng G. Long noncoding RNA derived from CD244 signaling epigenetically controls CD8+ T-cell immune responses in tuberculosis infection. Proc Natl Acad Sci U S A. 2015; 112:E3883-92. http://doi.org/10.1073/ pnas.1501662112.

17. Pawar K, Hanisch C, Palma Vera SE, Einspanier R, Sharbati S. Down regulated lncRNA MEG3 eliminates mycobacteria in macrophages via autophagy. Sci Rep. 2016; 6:19416. http://doi.org/10.1038/srep19416.

18. Fu Y, Xu X, Xue J, Duan W, Yi Z. Deregulated lncRNAs in B cells from patients with active tuberculosis. PLoS One. 2017; 12:e0170712. http://doi.org/10.1371/journal. pone. 0170712 .

19. Yang X, Yang J, Wang J, Wen Q, Wang H, He J, Hu S, He W, Du X, Liu S, Ma L. Microarray analysis of long noncoding RNA and mRNA expression profiles in human macrophages infected with Mycobacterium tuberculosis. Sci Rep. 2016; 6:38963. http://doi.org/10.1038/srep38963.

20. Zhao Z, Zhang M, Ying J, Hu X, Zhang J, Zhou Y, Zhou Y, Song X, Ying B. Significance of genetic polymorphisms in long non-coding RNA AC079767.4 in tuberculosis susceptibility and clinical phenotype in Western Chinese Han population. Sci Rep. 2017; 7:965. http://doi. org/10.1038/s41598-017-01163-y.

21. Wang Y. (2011). The compilation of the fifth national tuberculosis epidemiological sampling survey.

22. Gong J, Liu W, Zhang J, Miao X, Guo AY. lncRNASNP: a database of SNPs in lncRNAs and their potential functions in human and mouse. Nucleic Acids Res. 2015; 43:D181-6. http://doi.org/10.1093/nar/gku1000.

23. Yue B, Cai D, Liu C, Fang C, Yan D. LINC00152 functions as a competing endogenous RNA to confer oxaliplatin resistance and holds prognostic values in colon cancer. Mol Ther. 2016; 24:2064-77. http://doi.org/10.1038/ mt.2016.180.

24. Zhang YH, Fu J, Zhang ZJ, Ge CC, Yi Y. LncRNALINC00152 down-regulated by miR-376c-3p restricts viability and promotes apoptosis of colorectal cancer cells. Am J Transl Res. 2016; 8:5286-97.

25. Li J, Wang X, Tang J, Jiang R, Zhang W, Ji J, Sun B. HULC and LINC00152 act as novel biomarkers in predicting diagnosis of hepatocellular carcinoma. Cell Physiol Biochem. 2015; 37:687-96. http://doi. org/10.1159/000430387.

26. Cai Q, Wang Z, Wang S, Weng M, Zhou D, Li C, Wang J, Chen E, Quan Z. Long non-coding RNA LINC00152 
promotes gallbladder cancer metastasis and epithelialmesenchymal transition by regulating HIF-1alpha via miR138. Open Biol. 2017. http://doi.org/10.1098/rsob.160247.

27. Wagh V, Urhekar A, Modi D. Levels of microRNA miR16 and miR-155 are altered in serum of patients with tuberculosis and associate with responses to therapy. Tuberculosis (Edinb). 2017; 102:24-30. http://doi. org/10.1016/j.tube.2016.10.007.

28. Rajaram MV, Ni B, Morris JD, Brooks MN, Carlson TK, Bakthavachalu B, Schoenberg DR, Torrelles JB, Schlesinger LS. Mycobacterium tuberculosis lipomannan blocks TNF biosynthesis by regulating macrophage MAPKactivated protein kinase 2 (MK2) and microRNA miR-125b. Proc Natl Acad Sci U S A. 2011; 108:17408-13. http://doi. org/10.1073/pnas.1112660108.

29. Fu X, Zeng L, Liu Z, Ke X, Lei L, Li G. MicroRNA-206 regulates the secretion of inflammatory cytokines and MMP9 expression by targeting TIMP3 in Mycobacterium tuberculosis-infected THP-1 human macrophages. Biochem Biophys Res Commun. 2016; 477:167-73. http://doi. org/10.1016/j.bbrc.2016.06.038.

30. Wang C, Liu CM, Wei LL, Shi LY, Pan ZF, Mao LG, Wan XC, Ping ZP, Jiang TT, Chen ZL, Li ZJ, Li JC. A group of novel serum diagnostic biomarkers for multidrugresistant tuberculosis by iTRAQ-2D LC-MS/MS and solexa sequencing. Int J Biol Sci. 2016; 12:246-56. http://doi. org/10.7150/ijbs. 13805 .

31. Perwitasari DA, Atthobari J, Wilffert B. Pharmacogenetics of isoniazid-induced hepatotoxicity. Drug Metab Rev. 2015; 47:222-8. http://doi.org/10.3109/03602532.2014.984070.

32. Ramappa V, Aithal GP. Hepatotoxicity related to antituberculosis drugs: mechanisms and management. J Clin
Exp Hepatol. 2013; 3:37-49. http://doi.org/10.1016/j. jceh.2012.12.001.

33. Wu S, Wang YJ, Tang X, Wang Y, Wu J, Ji G, Zhang M, Chen G, Liu Q, Sandford AJ, He JQ. Genetic polymorphisms of glutathione S-transferase P1 (GSTP1) and the incidence of anti-tuberculosis drug-induced hepatotoxicity. PLoS One. 2016; 11:e0157478. http://doi. org/10.1371/journal.pone.0157478.

34. Huang YS, Chern HD, Su WJ, Wu JC, Lai SL, Yang SY, Chang FY, Lee SD. Polymorphism of the $\mathrm{N}$-acetyltransferase 2 gene as a susceptibility risk factor for antituberculosis drug-induced hepatitis. Hepatology. 2002; 35:883-9. http://doi.org/10.1053/jhep.2002.32102.

35. World Health Organization. (2010). Treatment of Tuberculosis: Guidelines. 4th edition, Geneva.

36. Benichou C. Criteria of drug-induced liver disorders. Report of an international consensus meeting. J Hepatol. 1990; 11:272-6.

37. Mehta RL, Awdishu L, Davenport A, Murray PT, Macedo E, Cerda J, Chakaravarthi R, Holden AL, Goldstein SL. Phenotype standardization for drug-induced kidney disease. Kidney Int. 2015; 88:226-34. http://doi.org/10.1038/ ki.2015.115.

38. Sotgiu G, Centis R, D’Ambrosio L, Spanevello A, Migliori GB; International Group for the study of Linezolid. Linezolid to treat extensively drug-resistant TB: retrospective data are confirmed by experimental evidence. Eur Respir J. 2013; 42:288-90. http://doi. org/10.1183/09031936.00191712. 